TITLE:

\title{
Separation of long DNA chains using a nonuniform electric field: A numerical study
}

AUTHOR(S):

Nagahiro, S; Kawano, S; Kotera, H

CITATION:

Nagahiro, S ... [et al]. Separation of long DNA chains using a nonuniform electric field: A numerical study. PHYSICAL REVIEW E 2007, 75(1):

011902.

ISSUE DATE:

2007-01

URL:

http://hdl.handle.net/2433/39918

RIGHT:

Copyright 2007 American Physical Society 
PHYSICAL REVIEW E 75, 011902 (2007)

\title{
Separation of long DNA chains using a nonuniform electric field: A numerical study
}

\author{
Shin-ichiro Nagahiro* \\ Department of Mechanical Engineering, Miyagi National College of Technology, Miyagi 981-1239, Japan \\ Satoyuki Kawano \\ Graduate School of Engineering Science, Osaka University, Osaka 560-8531, Japan \\ Hidetoshi Kotera \\ Graduate School of Engineering, Kyoto University, Kyoto 606-8501, Japan
}

(Received 22 August 2006; published 5 January 2007)

\begin{abstract}
In the present study, we investigate the migration of DNA molecules through a microchannel using a series of electric traps controlled by an ac electric field. We describe the motion of DNA based on Brownian dynamics simulations of a bead-spring chain. The DNA chain captured by an electric field escapes due to thermal fluctuation. The mobility of the DNA chain was determined to depend on the chain length, the mobility of which sharply increases when the length of the chain exceeds a critical value that is strongly affected by the amplitude of the applied ac field. Thus we can optimize the separation selectivity of the channel for DNA molecules that is to be separated, without changing the structure of the channel. In addition, we present a phenomenological description for the relationship between the critical chain length and the strength of binding electric field.
\end{abstract}

DOI: $10.1103 /$ PhysRevE.75.011902

PACS number(s): 87.15.Tt, 87.14.Gg, 87.15.Aa, 87.15.He

\section{INTRODUCTION}

Gel electrophoresis is commonly used in biological science to separate DNA molecules according to their size. Longer DNA molecules have a lower electrophoretic mobility in the random environment of a gel, and therefore separation could be performed using a dc electric field. However, gel electrophoresis has a lower efficiency for DNA molecules longer than about $20 k$ base pairs (bp), beyond which the length dependence of the mobility gradually disappears. In addition, pulsed-field electrophoresis has been widely used for separation of longer DNA molecules. This method also has a limit, with respect to both speed and size, with an upper limit of approximately $10 \mathrm{Mbp}$.

On the other hand, separation devices are required to be incorporated into a millimeter-sized chip with the development of highly integrated bioanalysis systems, micro-total analysis systems $(\mu$-TAS) or lab-on-a-chip devices $[1,2]$. However, introducing a gel into microchannels is a difficult problem due to the high viscosity involved. Furthermore, the gel-free system has an advantage in giving a well-defined microstructure for DNA separation devices. Therefore recently proposed gel-free separation techniques [3-8] have received considerable attention. Ajdari and Prost proposed a gel-free separation technique that uses free-flow electrophoresis together with a series of trapping by induced-dipole forces [3]. Based on their theoretical analysis they argued that, in their model, the mobility of a DNA chain monotonically decreases with size. More recently, Han and Craighead investigated a designed microchannel, which has alternating thin and thick regions [4-6], and found that longer DNA molecules were trapped in thick regions for a shorter time.

\footnotetext{
*Electronic address: nagahiro@miyagi-ct.ac.jp
}

Therefore shorter DNA molecules have lower mobilities. This has been confirmed both theoretically [9] and numerically [10]. Note that, as mentioned in Ref. [4], the free energy landscape of the above two systems are quite similar, the relation between mobility and DNA size shows a clear contrast. This discrepancy may originate from the fact that, in order to consider the escape of DNA chains from an electrode, Ajdari and Prost considered the chain as a point particle. In contrast, as observed experimentally, the deformation of DNA is important when a DNA molecule escapes from a thick region [4].

In the present paper, we revisit the Ajdari and Prost's model and investigate the mobility of DNA chains along the channel, taking the deformation of the DNA molecule into account. Our simulation verifies that longer DNA is trapped for a shorter time and the mobility increases suddenly when the chain exceeds a certain critical length. Furthermore, we found that this critical chain length is strongly affected by the voltage applied across the electrodes. Therefore we are able to adjust the regime in which the device well separates DNA chains without changing the structure of the channel. These numerical insights will help us to design practical separation devices in the future.

The present paper is organized as follows. In Sec. II, the proposed separation device and the simulation model are described. In Sec. III A, the results of our simulation are discussed, and in Sec. III B, a scaling analysis for the mobility of DNA chains is presented, the result of which are compared with the simulation results. In Sec. IV, we summarize the results obtained in the present study.

\section{SIMULATION MODEL}

For the present simulation, we adopt a bead-spring model, which represents a DNA molecule as $N$ beads or monomers 


\section{(a)}

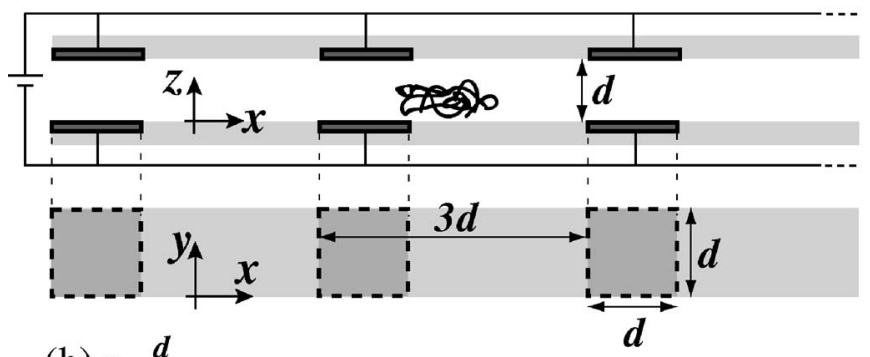

(b) $y=\frac{d}{2}$

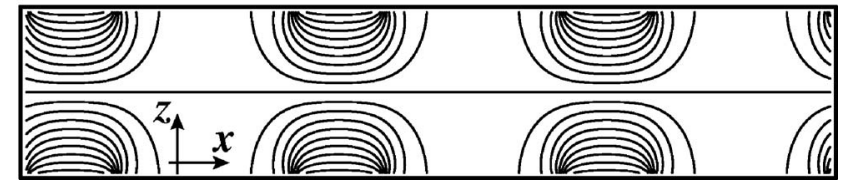

FIG. 1. (a) Schematic diagrams of a channel with electric traps in the $x y$ and $y z$ planes. The electrodes are square in shape and are placed along the channel at intervals of $3 d$. (b) The contour lines of the electric potential $\phi$ at the plane $y=d / 2$.

of diameter $\sigma$. Neighboring beads are connected by harmonic springs with constant $k$ as follows:

$$
\frac{V^{\mathrm{sp}}\left(r_{\mathrm{n}}\right)}{k_{\mathrm{B}} T}=\frac{1}{2} k\left(\frac{r_{\mathrm{n}}}{\sigma}\right)^{2},
$$

where $V^{\mathrm{sp}}$ is the potential energy of the spring, $r_{\mathrm{n}}$ is the distance between neighboring beads, $k_{\mathrm{B}}$ is the Boltzmann constant, and $T$ is the temperature. In order to take the excluded volume effect into account, all beads are assumed to interact with each other through the Weeks-ChandlerAndersen (WCA) potential,

$$
\frac{V^{\mathrm{WCA}}(r)}{k_{\mathrm{B}} T}=\left\{\begin{array}{cc}
\left(\frac{\sigma}{r}\right)^{12}-\left(\frac{\sigma}{r}\right)^{6}+\frac{1}{2}, & \frac{r}{\sigma}<2^{1 / 6} \\
0, & \text { otherwise }
\end{array}\right.
$$

where $r$ is the interbead distance.

Let us denote the thermal fluctuation force acting on the $i$ th bead as $\boldsymbol{\eta}_{i}(t)$. We assume that the viscous drag is proportional to the bead velocity with friction constant $\zeta$. A general theorem from statistical mechanics relates the random force and the friction constant as

$$
\left\langle\boldsymbol{\eta}_{i}(t) \boldsymbol{\eta}_{j}\left(t^{\prime}\right)\right\rangle=2 k_{\mathrm{B}} T \zeta \delta_{i j} \boldsymbol{I} \delta\left(t-t^{\prime}\right),
$$

where $\boldsymbol{I}$ is the $3 \times 3$ identity tensor, $\delta_{i j}$ is the Kronecker delta, and $\delta\left(t-t^{\prime}\right)$ is the delta function. The random force in our simulation is set so as to satisfy this relation in a discretized manner [11].

Schematic diagrams of the $x z$ and $x y$ planes of the channel are shown in Fig. 1. We simulate the motion of bead-spring chains inside this channel, the cross section of which is a square having a side length of $d$. For the $x$ direction, the periodic boundary condition with distance $L=9 d$ is assumed both for the electric field and the chain motion, unless mentioned specifically. The beads are assumed not to adhere to the channel wall. The WCA potential is also applied between the beads and the wall. In the corners of the channel, the two repulsive forces from different directions are summed.

Each bead carries an electric charge of $q_{\text {net }}$. For strongly charged polyelectrolytes such as DNA molecules, the charge $q_{\text {net }}$ should be regarded as the reduced charge due to counterion condensation. According to the Oosawa-Manning theory $[12,13]$, the charge of DNA, immersed in monovalent salt, is neutralized by $76 \%$. Hence for the beads representing $m$ base pairs, we set $q_{\text {net }} \simeq 2.0(1.0-0.76) m e^{-}=0.48 m e^{-}$, where $e^{-}$is the elementary electric charge.

Ajdari and Prost theoretically considered the onedimensional motion of a DNA molecule pulled by an electric field. In their model, a series of dielectric traps is placed by applying ac electric fields perpendicular to the direction of DNA migration. It is assumed that the frequency of the ac field is high enough that the molecule feels a static ratchetlike potential. In the present simulation, we set "parallelplate condensers" along the channel, as shown in Fig. 1(a), and apply a dc voltage across them. We also apply a uniform electric field $\boldsymbol{E}_{0}$ in the $x$ direction, which drives the DNA chain along the channel. The potential $\phi$ inside the channel is obtained by solving the Laplace equation $\nabla^{2} \phi=0$ with the boundary element method under the boundary conditions of $\phi= \pm \Delta U$ on the electrodes and $s \cdot \nabla \phi=0$ on the opposite wall, where $s$ is the surface normal. The potential $\phi$ obtained is approximately equivalent to that of Ajdari and Prost's channel in the $x$ direction [see Fig. 1(b)]. The electric force acting on a bead is given as $\boldsymbol{f}_{i}^{\mathrm{el}}=q_{\mathrm{net}}\left\{\boldsymbol{E}_{0}-\nabla \phi\left(\boldsymbol{r}_{i}\right)\right\}$, where $\boldsymbol{r}_{i}$ is the position of the $i$ th bead. Neglecting the inertia of the beads, we write the equation of motion for the $i$ th bead as follows:

$$
\zeta \dot{\boldsymbol{r}}_{i}=\boldsymbol{f}_{i}^{\mathrm{int}}+\boldsymbol{f}_{i}^{\mathrm{el}}+\boldsymbol{\eta}_{i}
$$

where $f_{i}^{\text {int }}$ represents the sum of the bead-bead and bead-wall interactions.

The natural units of our simulation are defined as follows. We use $\sigma$ as the unit length, $k_{\mathrm{B}} T$ as the unit energy, $\mathcal{E}$ $\equiv k_{\mathrm{B}} T / \sigma q_{\text {net }}$ as the unit electric field strength, and $\tau$ $=\zeta \sigma^{2} / k_{\mathrm{B}} T$ as the unit time. The channel size is $d=20 \sigma$. The spring constant $k$ is set to $10^{2}$, a condition in which the equilibrium length of the springs is $0.85 \sigma$ and no chain crossing occurs [14]. We integrated Eq. (4) with secondorder stochastic Runge-Kutta algorithms [15] using a time step of $\Delta t=1.75 \times 10^{-4} \tau$. The random force is represented as $\boldsymbol{\eta}_{i}=\left(k_{\mathrm{B}} T / \sigma\right) \sqrt{6 \tau / \Delta t} \boldsymbol{\psi}_{i}$, where $\boldsymbol{\psi}_{i}$ is a random vector that has independent components uniformly distributed on $[-1,1]$. We used Mersenne twister algorithms [16] to generate $\boldsymbol{\psi}_{i}$. The initial conformation of a chain is set by placing beads with an interval of $\sigma$ on a trajectory obtained from a threedimensional self-avoiding random walk.

Finally, we describe how the parameters of the proposed model can be related to those obtained experimentally. We first consider $\sigma=50 \mathrm{~nm}$, the order of the persistence length of DNA $[17,18]$, whereby one bead corresponds to 167 base pairs. The electric field $\mathcal{E}=65 \mathrm{~V} / \mathrm{cm}$ under the condition of $T=300 \mathrm{~K}$. Using the Stokes' formula $\zeta=6 \pi \sigma \eta$ with $\boldsymbol{\eta}$ as the viscosity of water, the time scale is estimated as $\tau=5.1$ $\times 10^{-4}$ sec. 


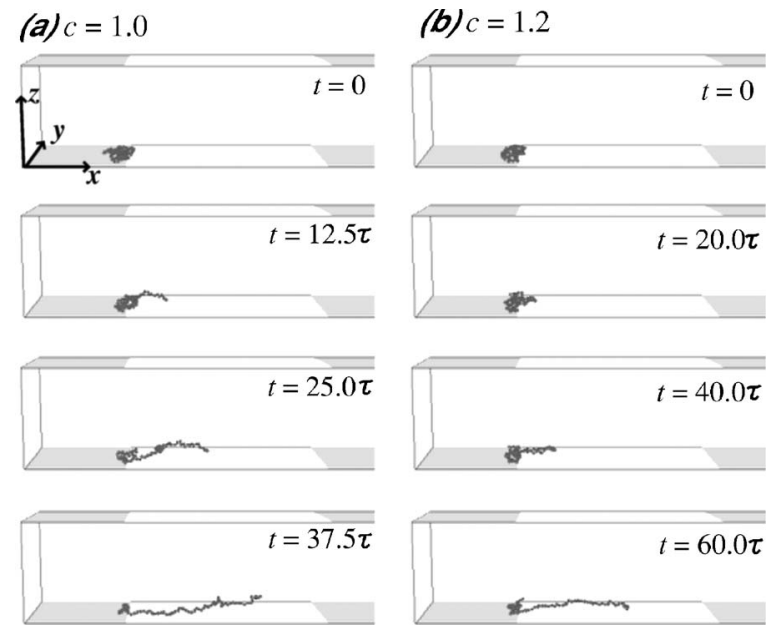

FIG. 2. A DNA chain of $N=80$ that escapes from an electric trap under the conditions of (a) $c=1.0$ and (b) $c=1.2$. The gray rectangular areas indicate electrodes.

The present model neglects the effect of counterions and hydrodynamic interactions. The adequacy of these simplifications was precisely discussed in Ref. [14]. This simulation model has successfully described the migration of DNA in gel $[19,20]$ and in a channel with entropic traps $[10,14]$. Therefore the model is thought to reproduce the experimental results with a few adjustable parameters.

\section{RESULTS AND DISCUSSION}

\section{A. Migration of DNA chain}

In this subsection, we describe the results of the present simulation for DNA migration in the microchannel. Here, we set $\boldsymbol{E}_{0}=\mathcal{E} \hat{\boldsymbol{x}}$, where $\hat{\boldsymbol{x}}$ is the unit vector parallel to the $x$ axis, and describe the applied voltage across each electrode with the dimensionless parameter $c=2 \Delta U / \mathcal{E} d$. The number of beads ranges from $N=5$ to $N=10^{3}$, which corresponds to DNA molecules from 0.8 to $170 \mathrm{kbp}$. We focus on the dimensionless mobility of a single isolated chain $\widetilde{\mu}=\mu \mathcal{E} \sigma / \tau$ as a function of $c$ and $N$. Figure 2 shows the snapshots of the present simulation, in which a chain of $N=80$ escapes from an electric trap under the conditions of $c=1.0$ and 1.2. Larger values of $c$ indicate stronger trapping by the transverse electric field. In this case, a $20 \%$ increase of $c$ roughly doubles the trapping lifetime of the DNA chain. The DNA chain is stretched during the migration to the next trap. The same behavior is also observed experimentally in the escape of DNA molecules from entropic traps [4,5]. Figure 3 shows the trajectories for chains of length $N=20,30, \ldots, 160$ at $c=1.0$. The flat regions of the trajectories indicate the trapping of chains on an electrode. Shorter chains tend to be trapped for longer times and longer chains $(N \geqslant 80)$ are only slightly affected by the traps. To evaluate mobility, we fit the trajectory of a chain that has passed through a trap ten times using a linear function. This slope corresponds to the dimensionless mobility $\tilde{\mu}$.

We show the relationship between $\tilde{\mu}$ and $N$ in Fig. 4. Each plot is the average of ten trials. For $c=0.8$, the mobility $\tilde{\mu}$

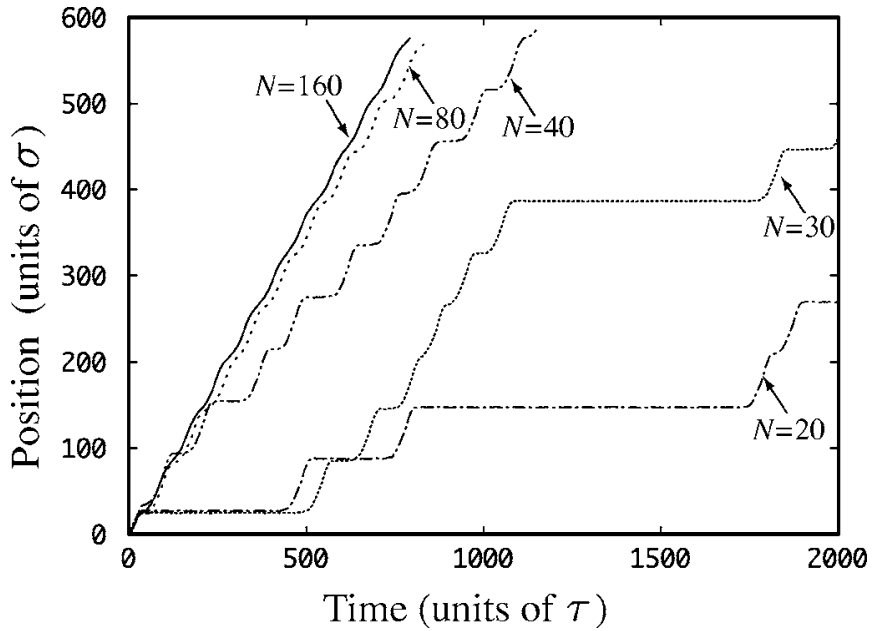

FIG. 3. Trajectories of DNA chains of $N=20,30, \ldots, 160$ with $c=1.0$. Only the $x$ component of the center of mass is plotted.

increases only gradually, and for $c \geqslant 1.0$, a sharp increase in mobility appears at a certain $N$. In the case of $c \geqslant 1.2$, short chains of less than $N \simeq 10^{2}$ are permanently trapped on an electrode and the resultant mobility is zero. The dispersion of the average tends to be large when the mobility shows the sudden increase. For the migration of long chains $N \simeq 800$ under the condition of $c=1.5$ and 1.7, the mobility again rises. This is because the chain is compressed by the side wall at $y=0$ and $d$, and stretched in the $x$ direction two or three times longer than the space between adjacent electrodes and thus the trapping by electrodes would be less efficient. In this regime, obtaining a theoretical understanding of the mobility is not easy, and so will be investigated in future studies. In the following subsection, we consider the dynamics of DNA chains trapped by a single electrode.

\section{B. Critical chain length $N_{\mathrm{c}}$}

The microchannel was efficient at separating DNA molecules in regions where the mobility $\tilde{\mu}$ depends strongly on chain length. Let us denote the critical chain length as $N_{c}$, at which the mobility increases suddenly. Figure 4 indicates that, by varying $c$, we can optimize the microchannel for the DNA molecules we would like to separate. In the following, we therefore consider the relationship between $N_{\mathrm{c}}$ and $c$.

Let us denote the trapping lifetime of DNA chains as $\tau_{t}(c, N)$. The dimensionless mobility can then be written as follows [4]:

$$
\tilde{\mu}=\frac{t_{0}}{t_{0}+\tau_{t}},
$$

where $t_{0}$ is the transit time between two adjacent electrodes. Figure 5(a) shows a schematic diagram of a bead-spring chain trapped on an electrode, in which $n$ beads of the chain have overcome an electric barrier $\Delta U$. To calculate the trapping lifetime $\tau_{t}$, we roughly estimate the change in total free energy $\Delta F(n)$ due to the escape of $n$ beads. When beads are trapped, their motion is restricted within a distance $\sigma$ from an electrode in $z$ direction. Thus we can characterize the $N-n$ 


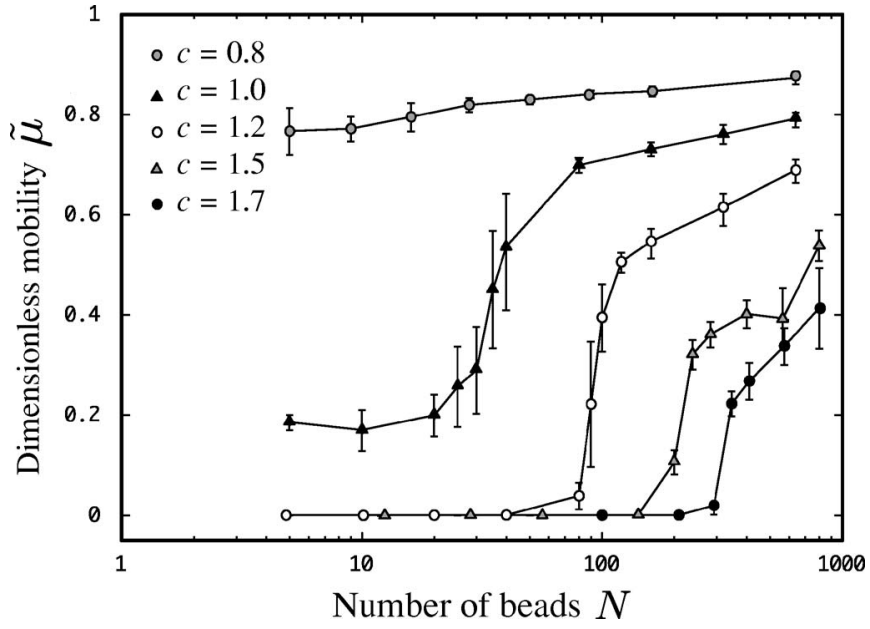

FIG. 4. Mobility $\tilde{\mu}$ of DNA chains for $c=0.8,1.0, \ldots, 1.7$ as a function of $N$. Each plot is the average and the error bars are drawn due to the standard deviations of ten trials.

beads on electrodes as having two typical lengths [21,22]:

$$
\begin{aligned}
& r_{g} \sim \sigma(N-n)^{\nu_{r}}, \\
& l_{g} \sim \sigma(N-n)^{\nu_{l}} .
\end{aligned}
$$

Unfortunately, we cannot analytically calculate the exponents $\nu_{r}$ and $\nu_{l}$. However, the present simulation evaluates $\nu_{r} \simeq 0.44$ and $\nu_{l} \simeq 0.37$. Each bead experiences the ratchetlike potential $V(x)$, as shown in Fig. 5(b), where we set $V(0)=0$. Near the right hand edge of the electrode, $V(x)$ can be written as

$$
V(x)=\left\{\begin{array}{cc}
-\mathcal{E} x & (x<0) \\
-\mathcal{E} x+\Delta U & (0<x)
\end{array}\right.
$$

To roughly estimate the electric energy of the DNA chains, we regard the chain as a one-dimensional system along the $x$ axis, and adopt a continuum approximation. The number density of beads projected on the $x$ axis is approximately given by

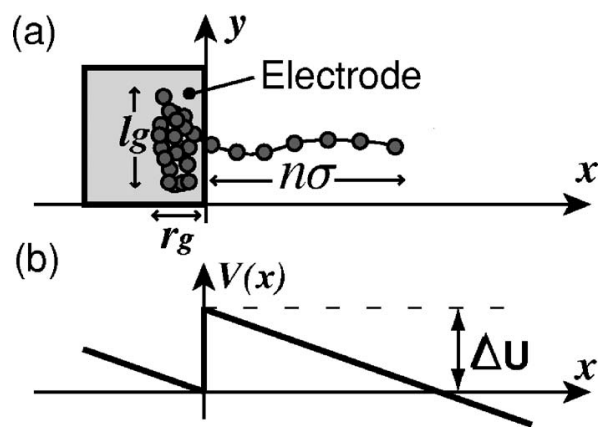

FIG. 5. (a) Schematic diagram of a bead-spring chain escaping from a trap. (b) Electric potential energy experienced by a bead.

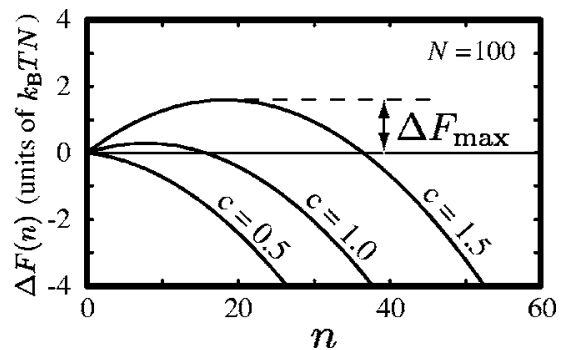

FIG. 6. Free energy difference $\Delta F(n)$ as a function of $n$ obtained from Eq. (10) with $A=3.4$.

$$
\rho(x)=\left\{\begin{array}{cc}
A l_{g} / \sigma^{2}, & \left(-r_{g}<x<0\right), \\
1 / \sigma, & (0<x<n \sigma), \\
0, & \text { otherwise },
\end{array}\right.
$$

where $A$ is a dimensionless constant. Then, the total electric potential energy of the chain $U(n)$ could be written as follows:

$$
\begin{aligned}
U(n) & \sim \int_{-r_{g}}^{n \sigma} q_{\text {net }} \rho(x) V(x) d x \\
& =A q_{\text {net }} \frac{l_{g} r_{g}^{2} \mathcal{E}}{2 \sigma^{2}}+n q_{\text {net }} \Delta U-\sigma q_{\text {net }} \mathcal{E} \frac{n^{2}}{2} \\
& =\frac{q_{\text {net }} \sigma \mathcal{E}}{2}\left\{A(N-n)^{\gamma}+\frac{c d}{\sigma} n-n^{2}\right\},
\end{aligned}
$$

where $\gamma \equiv 2 \nu_{r}+\nu_{l} \simeq 1.25$. Because the decrease in entropy due to the stretch of the beads is proportional to $n k_{B} T$, the total free energy difference $\Delta F(n)$ is given by

$$
\begin{aligned}
\frac{\Delta F(n)}{k_{\mathrm{B}} T} & \sim \frac{U(n)-U(0)}{k_{\mathrm{B}} T}+n \\
& =A\left\{(N-n)^{\gamma}-N^{\gamma}\right\}+\frac{c d}{2 \sigma} n-\frac{n^{2}}{2}+n .
\end{aligned}
$$

In Fig. 6, we plot $\Delta F(n)$ as a function of $n$ for $N=100$. For $c>1, \Delta F(n)$ increases at small $n$ and reaches a peak at $n=n^{*}$, whereas for $c=0.5, \Delta F(n)$ decreases monotonically with $n$. Let $\Delta F_{\max }$ be the maximum of $\Delta F(n)$. Trapping occurs when $d(\Delta F) /\left.d n\right|_{n=0}>0$, and $\tau_{t}$ is given by

$$
\tau_{t} \sim \exp \left(\Delta F_{\max } / k_{\mathrm{B}} T\right)
$$

We can calculate $\Delta F_{\max }$ by solving the following equation:

$$
\left.\frac{d}{d n} \frac{\Delta F(n)}{k_{\mathrm{B}} T}\right|_{n=n^{*}}=-A \gamma N^{\gamma-1}\left(1-\frac{n^{*}}{N}\right)^{\gamma-1}+\frac{c d}{2 \sigma}-n^{*}+1=0 .
$$

Here, we assume that the number of beads $N$ is much larger than $n^{*}$ and neglect the terms of order $N^{\gamma-2}$ and higher. We then obtain 


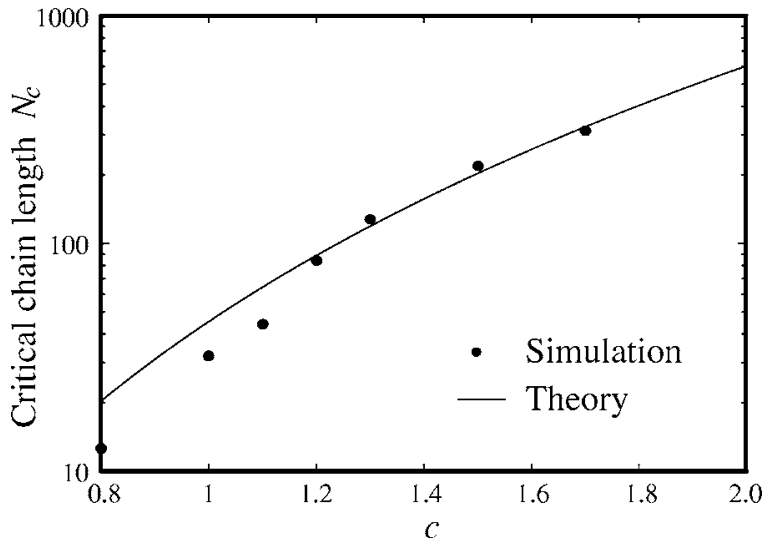

FIG. 7. Comparison of the critical chain length $N_{c}$ between the simulation result and the prediction of Eq. (14), where the black circles in Fig. 8 are estimated from the present simulation and the solid curve is a plot of Eq. (14) with parameter $A=3.4$.

$$
n^{*} \simeq \frac{c d}{2 \sigma}+1-A \gamma N^{\gamma-1}
$$

A sharp increase in $\tilde{\mu}$ appears when the free energy barrier is $\Delta F_{\max }=0$. From Fig. 5(b), this condition occurs only when $n^{*}=0$. We therefore obtain

$$
N_{c} \simeq\left(\frac{c d / 2+\sigma}{\sigma \gamma A}\right)^{1 /(\gamma-1)}, \quad \frac{1}{\gamma-1}=4.0
$$

with $A$ as a fitting parameter. In order to estimate the value of $N_{c}$ from Fig. 4, we consider linearly interpolated functions $\tilde{\mu}_{i n}(N)$, which is shown by the solid lines on the figure. Let $N_{i}(i=1,2,3, \ldots)$ be the point of interpolation. If $\tilde{\mu}_{i n}(N)$ has the maximum slope in $\left[N_{i}, N_{i+1}\right]$, we regard $\left(N_{i}+N_{i+1}\right) / 2$ as the critical length. Figure 7 shows that Eq. (14) is in good agreement with the present simulation with one adjustable parameter $A=3.4$. For $c \simeq 1$, Eq. (14) overestimates the simu- lation data because, in this region, the assumption $n^{*} \ll N$ is no longer valid.

\section{CONCLUDING REMARKS}

We have presented Brownian dynamics simulations of DNA migration in channels with electric trappings. The chain length dependence of mobility observed in the present simulation does not agree with the theory presented by $\mathrm{Aj}$ dari and Prost [3]. This discrepancy is concluded to be due to their simplification, in which DNA molecules escape from electric traps as point particles, because the numerical simulations of the present study demonstrated that the deformation of DNA chain is important when it escapes from the traps. The critical chain length $N_{c}$ is strongly affected by the voltage applied across the electrodes. Based on a phenomenological discussion, we found a simple scaling relationship between $N_{c}$ and the parameters of the present system, which agrees with the simulations.

The main advantage of the present channel is that one can control the critical chain length $N_{c}$ by changing the applied voltage of the condensers. However, the mobility of DNA decreases with the increase of the parameter $c$ as we see from the Fig. 4. This implies that the separation speed becomes lower when we apply a higher voltage to the condensers, which is required to separate the relatively long DNA chains. This drawback should be improved to design practical separation devices.

\section{ACKNOWLEDGMENTS}

One of the authors (S.N.) would like to thank Dr. Takahiro Sakaue for carefully reading the manuscript. S.N. also acknowledges the helpful discussions with Youhei Maruyama. This study was conducted as part of a joint research program, with the aim of developing a technological basis for COE in nano-medicine, which was carried out through the Kyoto City Collaboration of Regional Entities for Advancing Technology Excellence (CREATE) assigned by the Japan Science and technology Agency (JST).
[1] M. A. Burns et al., Science 282, 484 (1998).

[2] S. J. Lee and S. Y. Lee, Appl. Microbiol. Biotechnol. 64, 289 (2004).

[3] A. Ajdari and J. Prost, Proc. Natl. Acad. Sci. U.S.A. 88, 4468 (1991).

[4] J. Han, S. W. Turner, and H. G. Craighead, Phys. Rev. Lett. 83, 1688 (1999).

[5] J. Han and H. G. Craighead, Science 288, 1026 (2000).

[6] J. Han and H. G. Craighead, Anal. Chem. 74, 394 (2002).

[7] O. Bakajin, T. A. J. Duke, J. Tegenfeldt, C.-F. Chou, S. S. Chan, R. H. Austin, and E. C. Cox, Anal. Chem. 73, 6053 (2001)

[8] D. Schmalzing, L. Koutny, A. Adourian, P. Belgraderdagger, P. Matsudaira, and D. Ehrlich, Proc. Natl. Acad. Sci. U.S.A. 94, 10273 (1997).

[9] T. Sakaue, Eur. Phys. J. E 19, 477 (2006).

[10] M. Streek, F. Schmid, T. T. Duong, and A. Ros, J. Biotechnol. 112, 79 (2004)
[11] P. Grassia and E. J. Hinch, J. Fluid Mech. 308, 255 (1996).

[12] F. Oosawa, J. Polym. Sci. 13, 421 (1957).

[13] G. S. Manning, Q. Rev. Biophys. 11, 179 (1978).

[14] M. Streek, F. Schmid, T. T. Duong, D. Anselmetti, and A. Ros, Phys. Rev. E 71, 011905 (2005).

[15] R. L. Honeycutt, Phys. Rev. A 45, 600 (1992).

[16] M. Matsumoto and Y. Nishimura, ACM Trans. Model. Comput. Simul. 8, 3 (1998).

[17] P. J. Hagerman, Annu. Rev. Biophys. Biophys. Chem. 17, 265 (1988).

[18] K. Yoshikawa, Y. Matsuzawa, K. Minagawa, M. Doi, and M. Matsumoto, Biochem. Biophys. Res. Commun. 188, 1274 (1992).

[19] J. M. Deutsch, Phys. Rev. Lett. 59, 1255 (1987).

[20] H. Noguchi and M. Takasu, J. Chem. Phys. 114, 7260 (2001).

[21] P.-G. de Gennes, Scaling Concepts in Polymer Physics (Cornell University Press, Ithaca, NY, 1979).

[22] T. Sakaue and E. Raphaël, Macromolecules 39, 2621 (2006). 